Afr. J. Trad. CAM (2007) 4 (2): 173 - 184

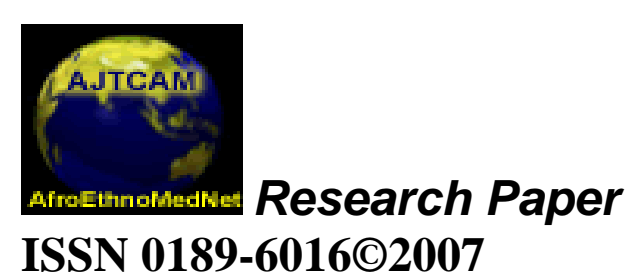
Afr. J. Traditional, Complementary and Alternative Medicines www.africanethnomedicines.net

\title{
ANTIMICROBIAL AND ANTIOXIDANT ACTIVITIES OF SOME NIGERIAN MEDICINAL PLANTS
}

\author{
A. J. Aladesanmi ${ }^{1}$, E. O. Iwalewa ${ }^{2}$, A. C. Adebajo ${ }^{1, *}$, E. O. Akinkunmi ${ }^{3}$, B. J.Taiwo ${ }^{4}$, F. O. \\ Olorunmola ${ }^{5}$ and A. Lamikanra ${ }^{3}$.
}

Departments of Pharmacognosy ${ }^{1}$, Pharmacology ${ }^{2}$, Pharmaceutics ${ }^{3}$, Pharmaceutical Chemistry ${ }^{4}$, and Drug Research and Production Unit ${ }^{5}$, Faculty of Pharmacy, Obafemi Awolowo University, Ile-Ife,

\begin{abstract} Osun State, Nigeria.

Ten Nigerian plants suggested from their ethnomedical uses to possess antimicrobial and antioxidant activities were studied for their anti-microbial and anti-oxidant properties. Antimicrobial activity was tested against Escherichia coli NCTC 10418, Pseudomonas aeruginosa, Staphylococcus aureus, Bacillus subtilis, Candida albicans, Candida pseudotropicalis and Trichophyton rubrum (clinical isolate). Trichilia heudelotti leaf extract showed both antibacterial and antifungal activities and was the most active against all the strains of bacteria tested. Boerhavia diffusa, Markhamia tomentosa and T. heudelotti leaf extracts inhibited the gram negative bacteria E.coli and P. aeruginosa strains whereas those of $M$. tomentosa, T. heudelotti and Sphenoceutrum jollyamum root inhibited at least one of the fungi tested. At a concentration of $312 \mu \mathrm{g} / \mathrm{ml}$, hexane and chloroform fractions of $T$. heudelotti extract inhibited 6 and $14 \%$ of the fifty mult-idrug resistant bacteria isolates from clinical infectins, respectively. At $\leq 5 \mathrm{mg} / \mathrm{ml}$, the $\mathrm{CHCl}_{3}(64 \%)$ and aqueous (22\%) fractions of $T$. heudelotti and those of $\mathrm{CHCl}_{3}$ (34\%) and EtOAC (48\%) of $M$. tomentosa gave the highest inhibition that was stronger than their corresponding methanol extracts. The corresponding $\mathrm{EC}_{50}$ of the extracts on M. acuminata, T. heudelotti, E. senegalensis and M. tomentosa were 4.00, 6.50, 13.33, and $16.50 \mathrm{ig} / \mathrm{ml}$ using the TLC staining and 1,1-dipheyl-2-picry-hydrazyl (DPPH) free radical scavenging assay. Therefore, leaf extracts of $M$. tomentosa and $T$. heudelotti, especially the latter, possess strong antimicrobial and antioxidant activities and should be further investigated. These activities justified the ethnomedical uses of these plants.
\end{abstract}

Key words: Antimicrobial, antifungal, antioxidant properties, Nigerian medicinal plants.

\section{Introduction}

Out of a total of 25 plants growing in Nigeria and selected based on their local uses, 15 of them have already been examined for their anti-microbial properties (Dalziel, 1956; Aladesanmi et al. 1986; Abbiw, 1989; Igbal et al., 1998; Kokuraro, 1993; Aderinokun et. al, 1999; Oluronke et al., 1999; Okafor et al., 2001; Wu et al., 2001) and hence were dropped. The remaining ten plants were investigated, some of which were used by the traditional healers for diseases such as diabetes, inflammation, loss of appetite, heart troubles, sores and skin infections (Oliver-Bever, 1986) as shown in Table 1. Eleven methanol extracts from these plants were studied for their antibacterial and antifungal properties against reference strains and multi-drug resistant clinical bacteria isolates. Their antioxidant activities were also assessed. This screening is of significant importance because of the urgent need for compounds that would be added to or replace the current antimicrobial agents to which microbes have become largely resistant (Chopra et. al., 1997).

\section{Materials and methods \\ Plant material and extracts preparation}

The dried (500 g) powder of the eleven plant parts was extracted with $\mathrm{MeOH}$ in a Soxhlet apparatus. The MeOH extracts of the 2 most antimicrobial active plants, $T$. heudelotti and M. tomentosa, were partitioned 
Adebajo et al., Afr. J. Trad. CAM (2007) 4 (2): 173 - 184

successively with n-hexane, $\mathrm{CHCl}_{3}$, EtOAc and $\mathrm{H}_{2} \mathrm{O}$ to obtain their corresponding fractions. Each extract or fraction $(1.0 \mathrm{~g})$ was taken to make stock solutions for the antimicrobial and antioxidant tests.

\section{Antimicrobial Activity \\ Disc Diffusion Assay (Murray et al., 1995)}

The strains used were; E.coli NCTC 10418, P. aeruginosa ATCC 10145, S. aureus NCTC 6571 and B. subtilis NCIB 3610 for bacteria and C. albicans, C. pseudotropicalis NCYC 6 and T. rubrum for fungi. The standard strains were from stocks of culture collections maintained in our laboratory. Bacteria were maintained on nutrient agar slants, and fungi on Sabouraud Dextrose agar slants at $4^{\circ} \mathrm{C}$ and subcultured monthly. Each extract (900 mg) was dissolved in $4 \mathrm{ml} \mathrm{MeOH} / \mathrm{H}_{2} \mathrm{O}$. Discs of Whatman No 1 filter paper $(\phi 6 \mathrm{~mm})$ were soaked with 2 drops of the extract using a sterile Pasteur pipette and allowed to dry at room temperature.

Two colonies of a 24-hour plate culture of each organism were transferred aseptically into $10 \mathrm{ml}$ sterile distilled water in a test tube and mixed thoroughly, using an electric shaker, for uniform distribution. A sterile cotton swab was then used to spread the resulting suspension uniformly on the surface of oven-dried Isosensitest agar (Oxoid) and Sabouraud dextrose agar plates (Sterillin) for bacteria and fungi, respectively. These were incubated for an hour at $37^{\circ} \mathrm{C}$ and $25^{\circ} \mathrm{C}$ for bacteria and fungi, respectively. Sterile forceps were used to place each of the discs on the agar plates aseptically and the plates were then refrigerated for $30 \mathrm{~min}$ at $4^{\circ} \mathrm{C}$ following which, the inoculated plates were incubated at $37^{\circ} \mathrm{C}$ for 24 hours for bacteria strains and at $25^{\circ} \mathrm{C}$ for 72 hours for the fungal strains. Antimicrobial activity was evaluated by noting the zone of inhibition against the test organisms. Those extracts showing any inhibition at all were noted for further tests for the quantitative assessment of their activity.

\section{Agar diffusion assay}

Dilutions of 40, 20, 10 and $5 \mathrm{mg} / \mathrm{ml}$ were prepared from $225 \mathrm{mg} / \mathrm{ml}$ stock solutions of the four extracts that inhibited the microorganisms. Volumes $(20 \mathrm{ml})$ of molten nutrient agar were seeded with $1 \mathrm{ml}$ portions of overnight cultures of microorganisms and poured into sterile Petri dishes $(85 \mathrm{~mm})$ and allowed to set. Holes of diameter $9 \mathrm{~mm}$ were made in the agar plates using a sterile metal cup-borer. Two drops of each extract and control were put in each hole under aseptic condition, kept at room temperature for 1 hour to allow the agents to diffuse into the agar medium and incubated accordingly (Reeves et al., 1979). Chloramphenicol $(100 \mu \mathrm{g} / \mathrm{ml})$ and acriflavine $(6.3 \mathrm{mg} / \mathrm{ml})$ were used as positive controls for bacteria and fungi respectively, $\mathrm{MeOH} / \mathrm{H}_{2} \mathrm{O}$ was the negative control. The plates were then accordingly incubated at $37^{\circ} \mathrm{C}$ for 24 hours for the bacterial strains and at $25^{\circ} \mathrm{C}$ for 72 hours for the fungal strains. The zones of inhibition were measured and extracts that gave significant activity against gram-negative isolates $(E$. coli and $P$. aeruginosa) were further tested against multidrug resistant bacteria including uropathogenic $E$. coli and $P$. aeruginosa wound isolates.

\section{Determination of the antibiotic susceptibility of bacteria isolates}

Forty-eight clinical isolates of bacteria from wound, stool and sputum of patients diagnosed with wound, gastro-intestinal tract and respiratory tract infections and apparently healthy individuals and two Bacillus species from the environment were used. The organisms were isolated on blood agar prepared with $5 \%$ human blood on Nutrient agar (Oxoid) and identified using their morphological characteristics and standard biochemical tests (Barrow and Feltham, 1993).

The Disc diffusion method (NCCLS, 2003) was used for the determination of microbial sensitivity. The antibiotic discs employed were: Nalidixic acid, Ofloxacin, Augmentin, Tetracycline, Nitrofurantoin, Chloramphenicol and Cephalothin all at $30 \mu \mathrm{g}$, Amoxycillin and Cotrimoxazole at $25 \mu \mathrm{g}$, Erythromycin at 15 $\mu \mathrm{g}$, Gentamicin, Ciprofloxacin and Penicillin-V at $10 \mu \mathrm{g}$. The zones of inhibition were measured and interpretation was in accordance with manufacturer's instructions (AB, Biodisc; PDM Interpretative chat).

Determination of Minimum Inhibitory Concentrations (MICs) of the fractions of $M$. tomentosa and T. heudelotti on multi drug resistant clinical isolates

The MIC were determined by the agar dilution method on Nutrient Agar (Oxoid) using a stock solution (50 mg/ml) of each fraction and extract dissolved in $50 \% \mathrm{MeOH} / \mathrm{H}_{2} \mathrm{O}$ (Murray et al., 1995). The final plate concentrations were $5.0,2.5,1.25,0.625$ and $0.3125 \mathrm{mg} / \mathrm{ml}$ for the extract/fractions and $0.5,0.25,0.125$, $0.0625,0.03125 \mathrm{mg} / \mathrm{ml}$ for chlorocresol (BDH), the +ve control. The hexane and $\mathrm{CHCl}_{3}$ fractions were dissolved in Tween 80 (Sigma-Aldrich Co., USA) and the rest in $50 \% \mathrm{MeOH} / \mathrm{H}_{2} \mathrm{O}$ and these vehicles were the -ve 
Adebajo et al., Afr. J. Trad. CAM (2007) 4 (2): 173 - 184

controls. Bacteria were grown for 18 hours in Nutrient broth (Oxoid) and culture suspensions of $10^{8} \mathrm{cfu} / \mathrm{ml}$ were applied to the surface of the nutrient agar plates containing dilutions of the fractions, chlorocresol, Tween 80 and methanol employing a multipoint inoculator. Plates were incubated at $37^{\circ} \mathrm{C}$ for 24 hrs, after which all plates were observed for growth. The minimum dilution of fractions completely inhibiting the growth of each organism was taken as the MIC.

\section{Antioxidant assay \\ Rapid radical scavenging screening}

With the aid of a capillary tube, stock solutions from the 10 extracts were spotted on silica gel thin layer chromatographic (TLC) plates and developed with the solvent systems of n-hexane 50:50, 30:70; $\mathrm{CHCl}_{3}$ 100\%; $\mathrm{CHCl}_{3}$-EtOAc 60:40; $\mathrm{CHCl}_{3}-\mathrm{MeOH}$ 80:20, 60:40; EtOAc-MeOH 90:10; EtOAc-MeOH-H${ }_{2} \mathrm{O}$ 100:17:13 and $\mathrm{BuOH}-\mathrm{AcOH}-\mathrm{H}_{2} \mathrm{O}$ 100:10:10, depending on the plant extract. After development, the chromatograms were dried and sprayed with a $0.4 \mathrm{mM}$ solution of the stable radical, DPPH. Yellow spots formed against purple background were taken as positive results. The time (duration) for the development of the yellow colour indicated whether the antioxidant activity was strong or not (Mensor et al., 2001; Burits and Bucar, 2000).

\section{DPPH photometric assay}

The free radical scavenging activities of each extract and ascorbic acid were analysed by the DPPH assay (Sanchez-Moreno, et al 1998). A $1.0 \mathrm{ml}$ of the test extract, at gradient final concentrations of 0.5 - 500 $\mu \mathrm{g} / \mathrm{ml}$, was mixed with $2 \mathrm{ml}$ of $0.3 \mathrm{mM} \mathrm{DPPH}$ solution in $\mathrm{MeOH}$ in a cuvette. The absorbance was taken at 517 $\mathrm{nm}$ after 20 minutes of incubation in the dark at room temperature. The experiment was done in triplicates. The percentage antioxidant activity was calculated as follows:

$\%$ Antioxidant Activity [AA $]=100-\left[\{(\right.$ Abs sample - Abs blank $) X 100\} /$ Abs $\left._{\text {control }}\right]$. Where Abs sample was the absorbance of sample solution $(2.0 \mathrm{ml})+$ DPPH solution $(1.0 \mathrm{ml}, 0.3 \mathrm{mM})$, Abs blank was the absorbance of Methanol $(1.0 \mathrm{ml})+$ sample solution $(2.0 \mathrm{ml})$, Abs control was the absorbance of DPPH solution $(1.0 \mathrm{ml}, 0.3 \mathrm{mM})$ + methanol $(2.0 \mathrm{ml})$.

\section{Results}

As shown in Table 2, among the 10 plants studied, only M. tomentosa and T. heudelotti leaf MeOH extracts showed both antifungal and antibacterial activities. The leaf $\mathrm{MeOH}$ extract of $B$. diffusa gave only antibacterial activity whereas that of $S$. jollyanum root showed only antifungal properties (Table 2). In addition, $T$. heudelotti extract showed significant activity against the gram-negative bacteria, $P$. aeruginosa (MIC = $10 \mathrm{mg} / \mathrm{ml}$ ) and $E$. coli $(\mathrm{MIC}=20 \mathrm{mg} / \mathrm{ml})$. The result of the susceptibility study showed a high level of antibiotic resistance by the clinical and environmental isolates (Table 3). There was a high level of antibiotic resistance to the commonly used antibiotics such as penicillin V (100\%), cephalothin (98\%), tetracycline (82\%), and augmentin (77\%). Some resistance was also demonstrated against fluoroquinolones e.g., ofloxacin (6.3\%), ciprofloxacin (21\%) and erythromycin (100\%). Generally, 64\% of the isolates were resistant to more than 50\% of the antibiotics tested (Table 3). The activity of the crude $\mathrm{MeOH}$ extracts and partition fractions of $M$. tomentosa and $T$. heudelotti against the multi-drug resistant isolates are given in Table 3 . At concentrations of $\leq$ $1.25 \mathrm{mg} / \mathrm{ml}$, the $\mathrm{CHCl}_{3}$ fractions of $M$. tomentosa and $T$. heudelotti showed the best activity, with the latter inhibiting the growth of most of the multi-drug resistant bacteria at a concentration of $312 \mu \mathrm{g} / \mathrm{ml}$. The intensity of the spots and reaction time of the DPPH radical scavenging capacities of the ten MeOH extracts using the TLC method showed that $M$. tomentosa, T. heudelotti and M. accuminata gave the highest antioxidant activities (Table 4). The $\mathrm{EC}_{50}$ of the extracts using DPPH photometric assay were M. acuminata 4.00, AA 4.65, T. heudelotti 6.50, E. senegalensis 13.33, M. tomentosa 16.50, P. barteri 66.67 and S. jollyamum $133.34 \mu \mathrm{g} / \mathrm{ml}$ (Figure 1).

\section{Discussion}

All the plants investigated have been reported to be used in ethnomedicine (Table 1).. Studies have been carried out to test the hypoglycaemic, antifungal and antioxidant activities of $B$. diffusa extracts (Chude et. al., 2001, Agrawal et. al., 2004, Amarnath and Pari, 2004). Similarly, Abo and Ashidi (1999) examined the antimicrobial and antifungal effects of the plant. Our results confirm the insignificant antimicrobial activity. As shown in Table 2, B. diffusa leaf extract has little activity against the bacteria and no activity against the fungi tested and therefore validates the traditional uses of the plant in treating boils and abscess. The root and leaf 
Adebajo et al., Afr. J. Trad. CAM (2007) 4 (2): 173 - 184

Table 1: Local Therapeutic uses of some Nigerian medicinal plants.

\begin{tabular}{|c|c|c|c|}
\hline Botanical Name (family) & $\begin{array}{l}\text { Local } \\
\text { Nigerian } \\
\text { Names }\end{array}$ & Plant part tested & Claimed therapeutic use \\
\hline $\begin{array}{l}\text { Boerhavia diffusa Linn. } \\
\text { (Nyctaginaceae) }\end{array}$ & Etiponla & Leaves & $\begin{array}{l}\text { Diabetes, anti inflammatory, Abscess, } \\
\text { boils }\end{array}$ \\
\hline $\begin{array}{l}\text { Ekebergia senegalansis A } \\
\text { Juss (Meliaceae) }\end{array}$ & $\begin{array}{l}\text { Ijebo, } \\
\text { Ayape, } \\
\text { oromu }\end{array}$ & Leaves & Antiepileptic, antimalaria \\
\hline $\begin{array}{l}\text { Gossypium arboretum } \\
\text { (Malvaceae) }\end{array}$ & Owu & Bark & Male contraceptive \\
\hline $\begin{array}{l}\text { Markhamia tomentosa (Benth) } \\
\text { K.Schum (Bignoniaceae) }\end{array}$ & Iru aya & Leaves & $\begin{array}{l}\text { Anti snake venom/bite, sore eyes, heart } \\
\text { pain, scrotal elephantiasis }\end{array}$ \\
\hline $\begin{array}{l}\text { Massularia acuminata (G. } \\
\text { Don) Bullock (Rubiaceae) }\end{array}$ & Pako Ijebu & Leaves & Cure of mouth infections \\
\hline $\begin{array}{l}\text { Pleioceras barteri Baill } \\
\text { (Apocynaceae) }\end{array}$ & Pariomo da & Root & Abortifacient, emmenagogue \\
\hline $\begin{array}{l}\text { Pleioceras barteri Baill } \\
\text { (Apocynaceae) }\end{array}$ & & Leaves & \\
\hline $\begin{array}{l}\text { Plumbago zeylanica Lin.- } \\
\text { Holl. (Plumbaginaceae) }\end{array}$ & Inabiri & Root & $\begin{array}{l}\text { Appetite stimulant, antiseptic skin } \\
\text { disease, scabies, ulcers. }\end{array}$ \\
\hline $\begin{array}{l}\text { Psidium guajava Lin.-Holl. } \\
\text { (Myrtaceae) }\end{array}$ & Guofa & Bark & Leaf used as antiseptic, antidiarrhoea. \\
\hline $\begin{array}{l}\text { Sphenoceutrum jollyanum } \\
\text { Pierre (Menispermaceae) }\end{array}$ & Akerejupon & Root & Chewing sticks, stomachic \\
\hline $\begin{array}{l}\text { Trichilia heudelotti (Oliver) } \\
\text { Planch (Meliaceae) }\end{array}$ & $\begin{array}{l}\text { Akorere, } \\
\text { rere }\end{array}$ & Leaves & Sores, heart troubles, pile \\
\hline
\end{tabular}


Table 2. In-vitro Antimicrobial activity of the methanolic extracts of some Nigerian medicinal Plants.

\begin{tabular}{|c|c|c|c|c|c|c|c|c|}
\hline \multirow[b]{2}{*}{ Extracts } & \multicolumn{8}{|c|}{ Diameter of zone of inhibition in mm exclusion of the $9 \mathrm{~mm}$ hole diameter } \\
\hline & $\begin{array}{l}\text { Concentration } \\
(\mathrm{mg} / \mathrm{ml})\end{array}$ & $\begin{array}{l}\text { E. coli NCTC } \\
10418 \text { (G - ve) }\end{array}$ & $\begin{array}{l}\text { B. subtilis NCIB } \\
3610 \text { (G + ve) }\end{array}$ & $\begin{array}{l}\text { P. aeruginosa. ATCC } \\
10145 \text { (G - ve) }\end{array}$ & $\begin{array}{l}\text { S. aureus NCTC6571 } \\
(\mathrm{G}+\mathrm{ve})\end{array}$ & C. albicans & C. pseudotropicalis & T. rubrum \\
\hline \multirow{5}{*}{$\begin{array}{l}\text { Boerhavia diffusa leaves } \\
\text { (methanol extract) }\end{array}$} & 5 & 0.0 & 0.0 & 0.0 & 0.0 & 0.0 & 0.0 & 0.0 \\
\hline & 10 & 0.0 & 0. & 0.0 & 1.0 & 0.0 & 0.0 & 0.0 \\
\hline & 20 & 0.01 & 0.0 & 0.0 & 1.0 & 0.0 & 0.0 & 0.0 \\
\hline & 40 & 0.0 & 2.0 & 0.0 & 2.0 & 0.0 & 0.0 & 0.0 \\
\hline & 225 & 0.0 & 6.0 & 3.0 & 4.0 & 0.0 & 0.0 & 0.0 \\
\hline \multirow{5}{*}{$\begin{array}{l}\text { Markhamia tomentosa } \\
\text { (methanol extract }\end{array}$} & 5 & 0.0 & 0.0 & 0.0 & 0.0 & 0.0 & 0.0 & 0.0 \\
\hline & 10 & 0.0 & 0.0 & 0.0 & 0.0 & 0.0 & 0.0 & 0.0 \\
\hline & 20 & 0.0 & 0.0 & 0.0 & 7.0 & 0.0 & 0.0 & 0.0 \\
\hline & 40 & 0.0 & 5.0 & 0.0 & 10.0 & 0.0 & 0.0 & 0.0 \\
\hline & 225 & 1.0 & 8.0 & 10.0 & 16.0 & 0.0 & 3.0 & 0.0 \\
\hline \multirow{5}{*}{$\begin{array}{l}\text { Sphenoceutrum jollyanum } \\
\text { Root (methanol extract) }\end{array}$} & 5 & 0.0 & 0.0 & 0.0 & 0.0 & 0.0 & 0.0 & 0.0 \\
\hline & 10 & 0.0 & 0.0 & 0.0 & 0.0 & 0.0 & 0.0 & 0.0 \\
\hline & 20 & 0.0 & 0.0 & 0.0 & 0.0 & 0.0 & 0.0 & 0.0 \\
\hline & 40 & 0.0 & 0.0 & 0.0 & 0.0 & 0.0 & 2.0 & 1.0 \\
\hline & 225 & 0.0 & 0.0 & 0.0 & 0.0 & 5.0 & 6.0 & 3.0 \\
\hline \multirow{5}{*}{$\begin{array}{l}\text { Trichila heudelotti leaves } \\
\text { (methanol extract) }\end{array}$} & 5 & 0.0 & 0.0 & 0.0 & 5.0 & 0.0 & 0.0 & 0.0 \\
\hline & 10 & 0.0 & 0.0 & 4.0 & 6.0 & 0.0 & 0.0 & 0.0 \\
\hline & 20 & 5.0 & 0.0 & 6.0 & 6.0 & 0.0 & 0.0 & 0.0 \\
\hline & 40 & 6.0 & 2.0 & 14.0 & 15.0 & 0.0 & 0.0 & 2.0 \\
\hline & 225 & 16.0 & 6.0 & 20.0 & 18.0 & 3.0 & 1.0 & 2.0 \\
\hline Chloramphenicol & $(100 \mu \mathrm{g} / \mathrm{ml})$ & 11.0 & 12.0 & 0.0 & 11.0 & N.T. & N.T. & N.T. \\
\hline Acriflavin & $6.3 \mathrm{mg} / \mathrm{ml}$ & N.T. & N.T. & N.T. & N.T. & 18.0 & 21.0 & 30.0 \\
\hline Methanol & 0.0 & 0.0 & 0.0 & 0.0 & 0.0 & 0.0 & 0.0 & 0.0 \\
\hline
\end{tabular}

The methanolic extracts of Ekebergia senegalansis leaf, Gossypium arboretum bark, Massularia acuminata leaf, Pleioceras barteri root and leaf, Plumbago zeylanica root and Psidium guajava bark were inactive to all the organisms tested. N.T.: Not tested. 


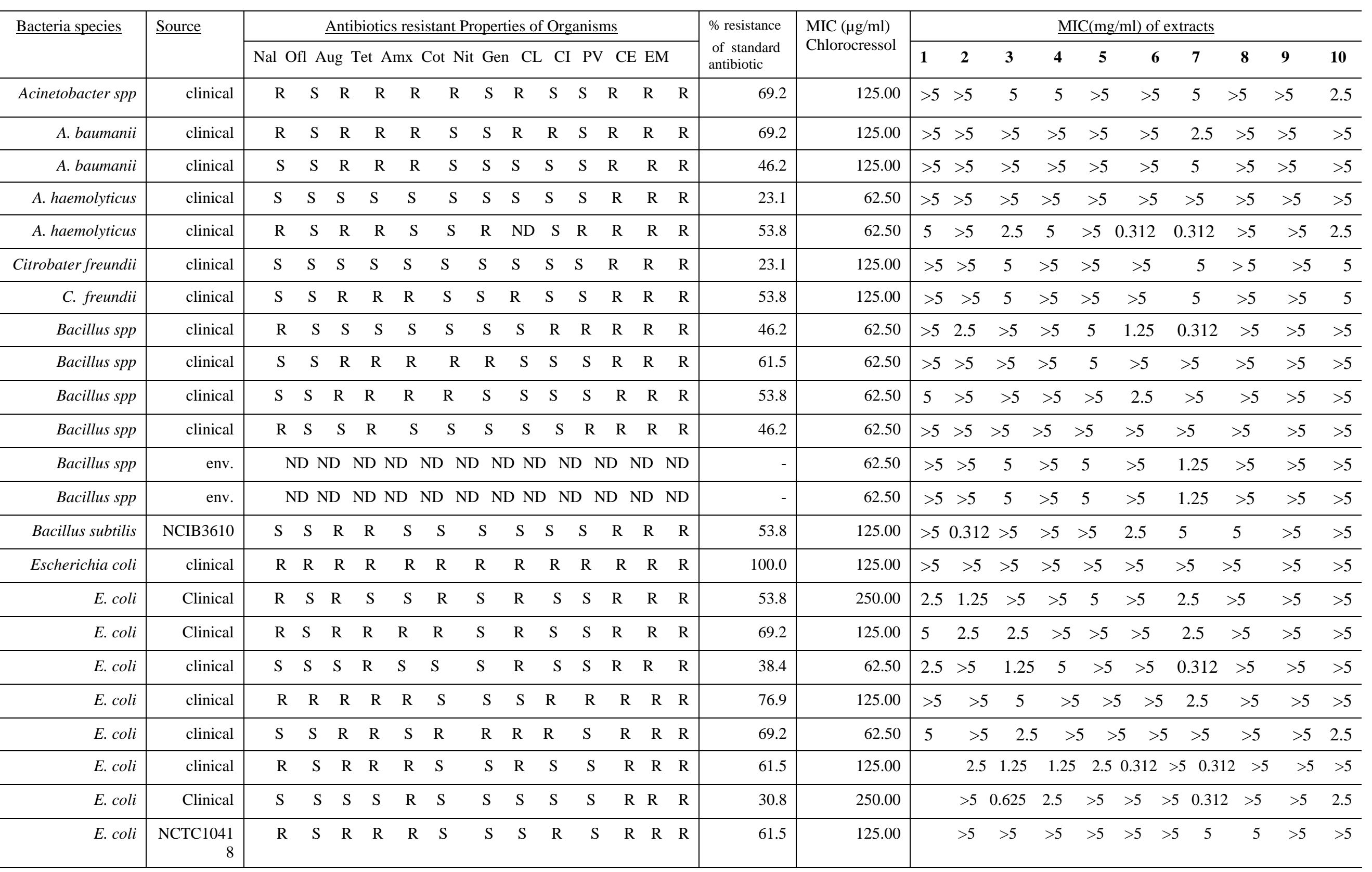




\begin{tabular}{|c|c|c|c|c|c|c|c|c|c|c|c|c|c|c|c|c|c|c|c|c|c|c|c|c|c|c|c|}
\hline Proteus vulgaris & Clinical & $\mathrm{S}$ & $S$ & $\mathrm{R}$ & $\mathrm{R}$ & $\mathrm{R}$ & $\mathrm{R}$ & & $S$ & $\mathrm{R}$ & $\mathrm{S}$ & $\mathrm{S}$ & $\mathrm{R}$ & $\mathrm{R}$ & $\mathrm{R}$ & 61.5 & 125.00 & $>5$ & 5 & $>5$ & & $2.5>5$ & $5>5$ & 1.25 & $>5$ & $>5$ & 2.5 \\
\hline P. vulgaris & Clinical & $\mathrm{S}$ & S & $\mathrm{R}$ & $\mathrm{R}$ & $\mathrm{R}$ & $S$ & S & $S$ & $\mathrm{R}$ & $\mathrm{S}$ & $\mathrm{S}$ & $\mathrm{R}$ & $\mathrm{R}$ & $\mathrm{R}$ & 53.8 & 125.00 & $>5$ & $>5$ & $>5$ & & $>5$ & $>5>5$ & $>5$ & $>5$ & $>5$ & $>5$ \\
\hline P. vulgaris & Clinical & $\mathrm{S}$ & $\mathrm{S}$ & $\mathrm{R}$ & $\mathrm{R}$ & $\mathrm{R}$ & $\mathrm{R}$ & $\mathrm{R}$ & $\mathrm{R}$ & $\mathrm{R}$ & $\mathrm{R}$ & $\mathrm{R}$ & $\mathrm{R}$ & $\mathrm{R}$ & $\mathrm{R}$ & 84.6 & 125.00 & $>5$ & 1.25 & $>5$ & & $>5>5$ & $5>5$ & $>5$ & $>5$ & $>5$ & $>5$ \\
\hline P. vulgaris & Clinical & $\mathrm{S}$ & S & $\mathrm{R}$ & $\mathrm{R}$ & $\mathrm{R}$ & $\mathrm{R}$ & $S$ & S & S & $\mathrm{S}$ & $\mathrm{S}$ & $\mathrm{R}$ & $\mathrm{R}$ & $\mathrm{R}$ & 53.8 & 125.00 & $>5$ & $>5$ & $>5$ & $>5$ & $5>5$ & 0.31 & 21.25 & $>5$ & $>5$ & 2.5 \\
\hline P. mirabilis & NCIB67 & $\mathrm{R}$ & $\mathrm{S}$ & $\mathrm{R}$ & $\mathrm{R}$ & $\mathrm{R}$ & $\mathrm{R}$ & $\mathrm{R}$ & & $\mathrm{R}$ & $\mathrm{R}$ & $\mathrm{R}$ & $\mathrm{R}$ & $\mathrm{R}$ & $\mathrm{R}$ & 92.3 & 125.00 & $>5$ & 2.5 & $>5$ & $>$ & $>5>5$ & 5 & 5 & $>5$ & $>5$ & $>5$ \\
\hline Pseudomonas spp & Clinical & $\mathrm{R}$ & $\mathrm{R}$ & $\mathrm{R}$ & $\mathrm{R}$ & $\mathrm{R}$ & $\mathrm{R}$ & $S$ & & $\mathrm{R}$ & $\mathrm{R}$ & $\mathrm{R}$ & $\mathrm{R}$ & $\mathrm{R}$ & $\mathrm{R}$ & 92.3 & 62.50 & $>5$ & 5 & 5 & $>5$ & $5>5$ & $>5$ & 2.5 & $>5$ & $>5$ & $>5$ \\
\hline Pseudomonas spp & Clinical & $\mathrm{S}$ & $\mathrm{S}$ & $\mathrm{R}$ & $\mathrm{R}$ & $\mathrm{S}$ & $\mathrm{S}$ & S & & S & S & $\mathrm{S}$ & $\mathrm{R}$ & $\mathrm{R}$ & $\mathrm{R}$ & 38.4 & 62.50 & $>5$ & $>5$ & 5 & $>5$ & $5>5$ & $>5$ & $>5$ & $>5$ & $>5$ & $>5$ \\
\hline Pseudomonas spp & Clinical & $\mathrm{R}$ & $\mathrm{S}$ & $\mathrm{R}$ & $\mathrm{R}$ & $\mathrm{R}$ & $\mathrm{R}$ & $\mathrm{R}$ & & $\mathrm{R} \quad \mathrm{I}$ & $\mathrm{R}$ & $\mathrm{S}$ & $\mathrm{R}$ & $\mathrm{R}$ & $\mathrm{R}$ & 84.6 & 62.50 & $>5$ & 1.25 & 5 & $>5$ & $5>5$ & $>5$ & 2.5 & $>5$ & $>5$ & $>5$ \\
\hline Pseudomonas spp & Clinical & $\mathrm{S}$ & $\mathrm{S}$ & $\mathrm{R}$ & $\mathrm{R}$ & $\mathrm{R}$ & $\mathrm{R}$ & S & & $S S$ & $S$ & $\mathrm{~S}$ & $\mathrm{R}$ & $\mathrm{R}$ & $\mathrm{S}$ & 46.2 & 500.00 & $>5$ & $>5$ & 5 & $>5$ & $5>5$ & $>5$ & $>5$ & $>5$ & $>5$ & $>5$ \\
\hline $\begin{array}{r}\text { Pseudomonas } \\
\text { aeruginosa }\end{array}$ & $\begin{array}{r}\text { ATCC1014 } \\
5\end{array}$ & $\mathrm{R}$ & $S$ & S & $\mathrm{S}$ & S & $\mathrm{S}$ & $\mathrm{S}$ & & S & $S$ & $\mathrm{~S}$ & $\mathrm{R}$ & $\mathrm{R}$ & $\mathrm{R}$ & 30.8 & 500.00 & $>5$ & 5 & 5 & $>5$ & $5>5$ & $>5$ & 5 & $>5$ & $>5$ & $>5$ \\
\hline Salmonella spp & Clinical & $\mathrm{R}$ & S & $\mathrm{R}$ & $\mathrm{R}$ & $\mathrm{R}$ & $\mathrm{S}$ & S & & $\mathrm{R}$ & S & S & $\mathrm{R}$ & $\mathrm{R}$ & $\mathrm{R}$ & 61.5 & 125.00 & $>5$ & $>5$ & $>5$ & $>5$ & 5 & $>5$ & $>5$ & $>5$ & $>5$ & $>5$ \\
\hline Salmonella spp & Clinical & $\mathrm{R}$ & $\mathrm{S}$ & $\mathrm{R}$ & $\mathrm{R}$ & $\mathrm{R}$ & $\mathrm{R}$ & $\mathrm{S}$ & & S & S & S & $\mathrm{R}$ & $\mathrm{R}$ & $\mathrm{R}$ & 61.5 & 125.00 & $>5$ & $>5$ & $>5$ & & $>5 \quad 5$ & $>5$ & $>5$ & $>5$ & $>5$ & $>5$ \\
\hline Salmonella spp & Clinical & $\mathrm{S}$ & $\mathrm{S}$ & S & $\mathrm{R}$ & $\mathrm{S}$ & $\mathrm{R}$ & $\mathrm{S}$ & & $\mathrm{R}$ & $\mathrm{R}$ & S & $\mathrm{R}$ & $\mathrm{R}$ & $\mathrm{R}$ & 53.8 & 125.00 & $>5$ & $>5$ & $>5$ & & $>5$ & $>5$ & $5>5$ & $>5$ & $>5$ & $>5$ \\
\hline Salmonella spp & Clinical & $\mathrm{R}$ & $\mathrm{S}$ & $\mathrm{R}$ & $\mathrm{R}$ & $\mathrm{R}$ & $\mathrm{S}$ & $\mathrm{R}$ & $\mathrm{R}$ & $\mathrm{R}$ & $\mathrm{R}$ & $S$ & $\mathrm{R}$ & $\mathrm{R}$ & $\mathrm{R}$ & 76.9 & 125.00 & $>5$ & $>5$ & $>5$ & & 5 & $>5$ & $5>5$ & $>5$ & $>5$ & $>5$ \\
\hline $\begin{array}{r}\text { Salmonella enterica } \\
\text { var. choleraesius }\end{array}$ & Clinical & $\mathrm{S}$ & $\mathrm{S}$ & $\mathrm{S}$ & $\mathrm{S}$ & S & S & S & & $S$ & S & $S$ & $\mathrm{R}$ & $\mathrm{S}$ & $\mathrm{R}$ & 15.4 & 62.50 & 2.5 & $>5$ & 1.25 & 1.2 & 25 & $>5$ & 0.625 & $>5$ & 2.5 & 2.5 \\
\hline $\begin{array}{r}\text { Salmonella enterica } \\
\text { var. typhimurium }\end{array}$ & Clinical & $\mathrm{S}$ & $\mathrm{S}$ & $\mathrm{R}$ & $\mathrm{R}$ & S & S & $\mathrm{S}$ & S & $S$ & S & S & $\mathrm{R}$ & $\mathrm{R}$ & $\mathrm{R}$ & 38.4 & 125.00 & $>5$ & $>5$ & $>5$ & $>5$ & $5>5$ & $>5$ & 5 & $>5$ & $>5$ & $>5$ \\
\hline $\begin{array}{r}\text { Staphylococcus } \\
\text { aureus }\end{array}$ & Clinical & $\mathrm{S}$ & S & $\mathrm{R}$ & $\mathrm{R}$ & $\mathrm{R}$ & $\mathrm{R}$ & $\mathrm{S}$ & $\mathrm{R}$ & R & S & $\mathrm{S}$ & $\mathrm{R}$ & $\mathrm{R}$ & $\mathrm{R}$ & 61.5 & 62.50 & $>5$ & $>5$ & 5 & $>5$ & $5>5$ & $5>5$ & 2.5 & $>5$ & $>5$ & $>5$ \\
\hline S. aureus & Clinical & $\mathrm{S}$ & $\mathrm{S}$ & $\mathrm{R}$ & $\mathrm{R}$ & $\mathrm{R}$ & $\mathrm{R}$ & $\mathrm{R}$ & $\mathrm{R}$ & $\mathrm{R}$ & & $\mathrm{S}$ & $\mathrm{R}$ & $\mathrm{R}$ & $\mathrm{R}$ & 76.9 & 62.50 & $>5$ & $>5$ & $>5$ & $>5$ & $>5$ & $>5$ & 2.5 & $>5$ & $>5$ & $>5$ \\
\hline S. aureus & Clinical & $\mathrm{S}$ & $\mathrm{S}$ & $\mathrm{R}$ & $\mathrm{R}$ & $\mathrm{R}$ & $\mathrm{R}$ & $\mathrm{S}$ & $\mathrm{R}$ & S & & $\mathrm{S}$ & $\mathrm{R}$ & $\mathrm{R}$ & $\mathrm{R}$ & 61.5 & 62.50 & $>5$ & $>5$ & $>5$ & $>5$ & $5>5$ & $>5$ & $>5$ & $>5$ & $>5$ & $>5$ \\
\hline S. aureus & Clinical & $\mathrm{R}$ & $\mathrm{S}$ & $\mathrm{R}$ & $\mathrm{R}$ & $\mathrm{S}$ & $\mathrm{S}$ & $\mathrm{S}$ & $\mathrm{R}$ & $\mathrm{R}$ & & $\mathrm{R}$ & $\mathrm{R}$ & $\mathrm{R}$ & $\mathrm{R}$ & 69.2 & 62.50 & $>51$ & 1.25 & $>5$ & $>5$ & 5 & $>5$ & 0.312 & $>5$ & $>5$ & $>5$ \\
\hline S. aureus & Clinical & $\mathrm{R}$ & $\mathrm{S}$ & $\mathrm{R}$ & $\mathrm{R}$ & $\mathrm{R}$ & $\mathrm{R}$ & $\mathrm{S}$ & $\mathrm{S}$ & S & $S$ & $\mathrm{~S}$ & $\mathrm{R}$ & $\mathrm{R}$ & $\mathrm{R}$ & 61.5 & 62.50 & $>5$ & $>5$ & $>5$ & $>5$ & $>5$ & $>5$ & $>5$ & $>5$ & $>5$ & $>5$ \\
\hline S. aureus & Clinical & $\mathrm{S}$ & $\mathrm{S}$ & $\mathrm{R}$ & S & $\mathrm{S}$ & $\mathrm{R}$ & $\mathrm{S}$ & $\mathrm{R}$ & S & & S & $\mathrm{R}$ & $\mathrm{R}$ & $\mathrm{R}$ & 46.2 & 62.50 & $>5$ & $>5$ & 5 & $>5$ & $>5$ & $>5$ & 2.5 & $>5$ & $>5$ & 2.5 \\
\hline S. aureus & Clinical & $\mathrm{R}$ & $\mathrm{S}$ & $\mathrm{S}$ & $\mathrm{R}$ & $\mathrm{S}$ & $\mathrm{S}$ & $\mathrm{S}$ & $\mathrm{R}$ & S & & S & $\mathrm{R}$ & $\mathrm{R}$ & $\mathrm{R}$ & 46.2 & 62.50 & 1.25 & 1.25 & 1.25 & $>5$ & $>5$ & $>5$ & 0.312 & $>5$ & $>5$ & 2.5 \\
\hline S. aureus & NCTC6571 & $\mathrm{S}$ & $\mathrm{S}$ & S & $\mathrm{S}$ & $\mathrm{S}$ & $\mathrm{S}$ & S & $\mathrm{S}$ & $\mathrm{S}$ & & S & $\mathrm{R}$ & $\mathrm{R}$ & $\mathrm{R}$ & 23.1 & 125.00 & 1.25 & $5>5$ & 1.25 & 5 & $>5$ & 0.312 & 5 & $>5$ & $>5$ & $>5$ \\
\hline $\begin{array}{r}\text { Staphylococcus } \\
\text { epidermidis }\end{array}$ & clinical & $\mathrm{R}$ & $\mathrm{S}$ & $\mathrm{R}$ & $\mathrm{R}$ & $\mathrm{R}$ & $\mathrm{R}$ & $\mathrm{S}$ & $\mathrm{R}$ & S & & $\mathrm{R}$ & $\mathrm{R}$ & $\mathrm{R}$ & $\mathrm{R}$ & 76.9 & 62.50 & 1.25 & 0.625 & 2.5 & $>5$ & 1.25 & 0.625 & 1.25 & $>5$ & $>5$ & $>5$ \\
\hline S. epidermidis & clinical & $\mathrm{S}$ & $\mathrm{S}$ & $\mathrm{R}$ & $\mathrm{R}$ & $\mathrm{R}$ & $\mathrm{R}$ & $\mathrm{S}$ & $\mathrm{R}$ & $\mathrm{R}$ & & $\mathrm{S}$ & $\mathrm{R}$ & $\mathrm{R}$ & $\mathrm{R}$ & 69.2 & 62.50 & 1.25 & 0.625 & 2.5 & $>5$ & 1.25 & 0.625 & 1.25 & $>5$ & $>5$ & $>5$ \\
\hline Vibrio cholerae & clinical & $\mathrm{R}$ & $\mathrm{S}$ & $\mathrm{R}$ & $\mathrm{R}$ & $\mathrm{R}$ & $\mathrm{S}$ & $\mathrm{S}$ & $\mathrm{R}$ & $S$ & & $\mathrm{~S}$ & $\mathrm{R}$ & $\mathrm{R}$ & $\mathrm{R}$ & 61.5 & 500.00 & $>5$ & $>5$ & 5 & $>5$ & $>5$ & $>5$ & 1.25 & $>5$ & $>5$ & $>5$ \\
\hline
\end{tabular}

Keys: 1, 2, 3, 5: n-hexane, chloroform, ethylacetate and aqueous partition fraction of Markhamia tomentosa methanol (MeOH) extract; 4: MeOH extract of M. tomentosa;

6, 7, 8, 10: n-hexane, chloroform, ethylacetate and aqueous partition fraction of Trichilia heudelotti methanol (MeOH) extract; 9: MeOH extract of T. heudelotti;

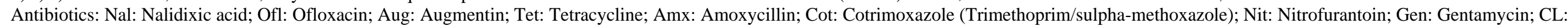
Chloramphenicol; CI: Ciprofloxacin; PV: Penicillin V; CE: Cephalothin; EM: Erythromycin. R: Resistant; S: Sensitive; ND: Not Determined 
Adebajo et al., Afr. J. Trad. CAM (2007) 4 (2): 173 - 184

Table 4: Radical scavenging abilities of the methanolic extracts from Ten Nigerian medicinal plants using rapid DPPH TLC screening.

\begin{tabular}{|l|l|l|}
\hline Plants (parts) & Reaction speed & Intensity of spots \\
\hline Sphenocentrium jollyanum (root) & Slow & ++ \\
\hline Pleioceras barteri (root) & Slow & ++ \\
\hline Pleioceras barteri (leaf) & - & - \\
\hline Massularia accuminata (leaf) & Fast & +++ \\
\hline Boerhavia diffusa (leaf) & Slow & + \\
\hline Plumbago raylanica (root) & - & - \\
\hline Markhamia tomentosa (leaf) & Fast & +++ \\
\hline Gossypium arberum (stem) & - & - \\
\hline Ekebergia senegalensis (leaf) & Slow & ++ \\
\hline Trichilia heudoleotii (leaf) & Fast & +++ \\
\hline
\end{tabular}

Keys: $\quad$-: no yellow colouration,

+ : weak intensity of yellow colouration (15 - 30 mins before colour development)

++ : intermediate intensity ( $1-15$ mins before colour development)

+++ : strong intensity (immediate reaction)

extracts of S. jollyanum have antiviral properties (Moody and Roberts, 2002a, b), the extracts of various morphological parts possess significant antioxidant, in-vitro antipyretic and analgesic activities (Oke and Hamburger, 2002; Mutho et al., 1998). The present results suggest that $S$. jollyanum possess significant antifungal activity (Table 2). Thus, this plant could be a source of useful antifungal agent. The other six plants investigated (Table 2) did not show any significant antibacterial or antifungal activity; however, activity has been reported for the Ethiopian $P$. zeylanica root on some pneumonia causing pathogens (Lemma et al., 2002).

The observation of weak and moderate antioxidant activities of B. diffusa and S. jollyanum extracts (Table 4) is in consistent with those reported (Mutho et al., 1998; Chude et. al., 2001, Oke and Hamburger, 2002; Agrawal et. al., 2004, Amarnath and Pari, 2004). The leaf extract of $M$. tomentosa showed good anti-staphylococcal activity as well as activities against $P$. aeruginosa and $B$. subtilis. It also showed encouraging activities against the fungus $C$. pseudotropicalis thereby demonstrating activity against a broad spectrum of organisms (Table 2). Extract of $T$. heudelotti leaves showed better antifungal and antibacterial activities than $M$. tomentosa (Table 2). T. heudelotti revealed an interesting activity against $S$. aureus $(\mathrm{MIC}=5 \mathrm{mg} / \mathrm{ml}), P$. aeruginosa $(\mathrm{MIC}=10 \mathrm{mg} / \mathrm{ml})$ and some activities against $E$. coli $(\mathrm{MIC}=20 \mathrm{mg} / \mathrm{ml}), B$. subtilis and $T$. rubrum $(\mathrm{MIC}=40 \mathrm{mg} / \mathrm{ml})$. Leaf extract of $T$. heudelotti also showed weak activities against the remaining 2 fungi tested (Table 2). 
Adebajo et al., Afr. J. Trad. CAM (2007) 4 (2): 173 - 184

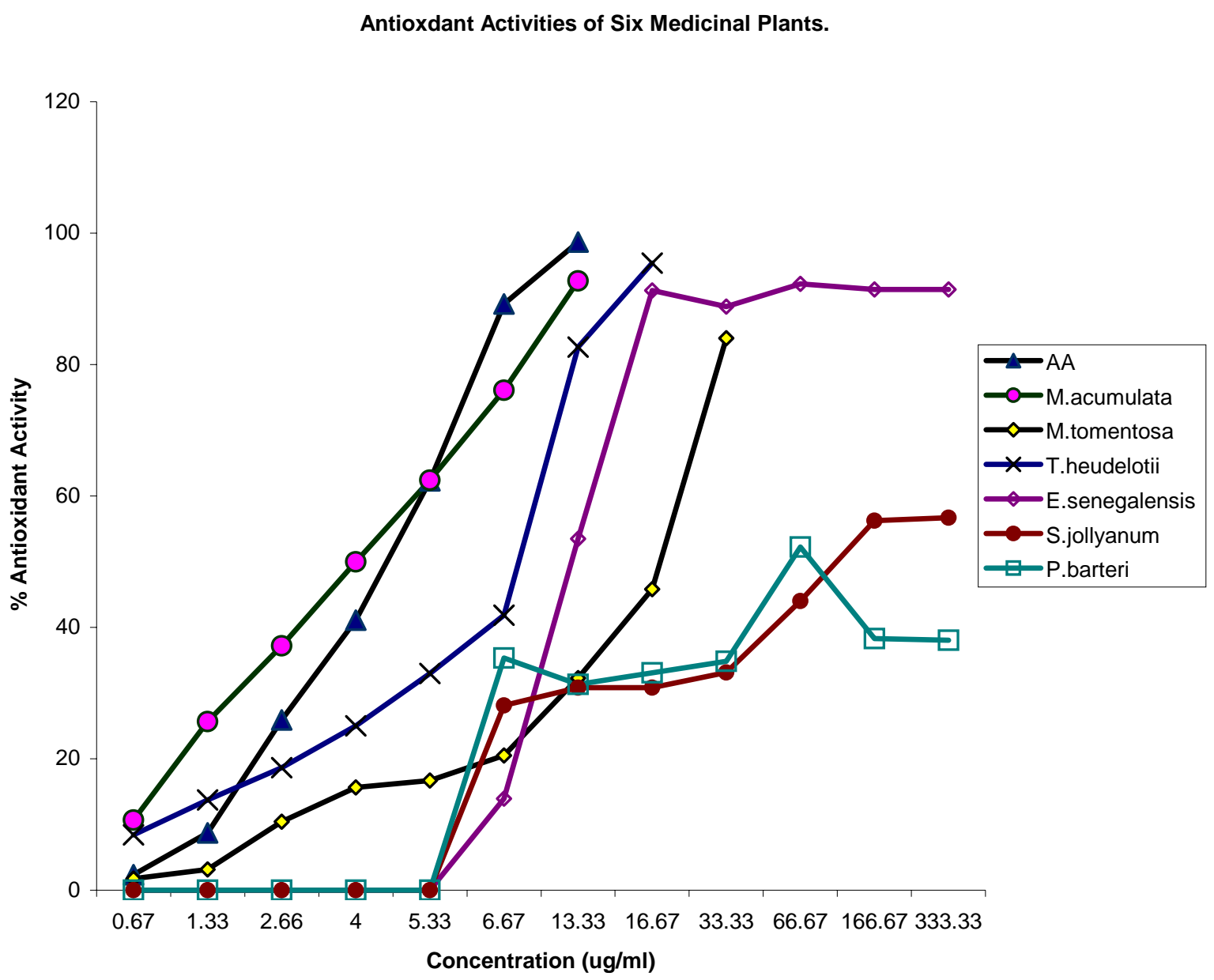

Figure 1: Antioxidant activity of six medicinal plants

The extracts of the two most active plants M. tomentosa and T. heudelotti were further fractionated to ascertain the properties of the active ingredients. These were tested against 50 multidrug resistant isolates from clinical and environmental sources including the organisms from the family Enterobacteriaceae which are commonly involved in clinical infections and are known to be highly versatile at acquiring resistance characteristics (Lamikanra et al., 1989). Two versatile pathogens, $P$. aeruginosa, a major nosocomial pathogen with low intrinsic susceptibility to antimicrobial agents and very high ability to acquire resistance, and $S$. aureus were also among those tested The organisms studied covered gram positive and gram-negative isolates (Table 3) and the demonstrated activities of the fractions showed that these plants have a wide spectrum of activity.

The bacteria used in Table 3 were not only resistant to the routinely used antibiotics in Nigeria such as Penicillin V and Erythromycin (100\%); Cephalothin (98\%), Tetracycline (82\%), Augmentin (77\%) and Cotrimoxazole but also showed resistance to the newer generation antibiotics like the fluoroquinolones e.g. ofloxacin (6.3\%) and ciprofloxacin (21\%). Sixty-four (64\%) percent of the isolates was resistant to more than $50 \%$ of the antibiotics tested (Table 3). The resistance properties of these isolates showed the worsening situation of antibiotics resistance in the Nigerian environment and lead credence to the search for substances that could be added to or replace the antibiotics in current clinical use, which are becoming less useful with every 
Adebajo et al., Afr. J. Trad. CAM (2007) 4 (2): 173 - 184

passing hour (Chopra et al., 1997, Okeke and Sosa, 2003). The better activities observed for the extracts and fractions of the two most active plants may provide an answer to this phenomenon.

The partition fractions, n-hexane, $\mathrm{CHCl}_{3}$, EtOAc, $\mathrm{H}_{2} \mathrm{O}$ together with the very active crude $\mathrm{MeOH}$ extracts of $M$. tomentos $a$ and $T$. heudelotti showed MIC of $<5 \mathrm{mg} / \mathrm{ml}$ against clinical and environmental isolates, especially against microbes that showed $>60 \%$ resistance against commonly used antibiotics (Table 3 ). Hence, extracts and active fractions of these plants may therefore be considered as alternative antimicrobial agent(s) against possible multiple antibiotic resistant strains that are widespread in the communities, especially in ethnomedicine (Chambers, 2003). At $\leq 1.25 \mathrm{mg} / \mathrm{ml}$, the orders of activity presented for the fractions and extracts of $M$. tomentosa and $T$. heudelotti were $\mathrm{MeOH}<$ n-hexane $=\mathrm{H}_{2} \mathrm{O}<\mathrm{EtOAc}^{2}<\mathrm{CHCl}_{3}$; and $\mathrm{EtOAc}=\mathrm{MeOH}=$ $\mathrm{H}_{2} \mathrm{O}<$ n-hexane $<\mathrm{CHCl}_{3}$ respectively (Table 3). The better activities of the fractions over the mother $\mathrm{MeOH}$ extracts showed that definite compounds present in these fractions were responsible for these activities. The $M$. tomentosa and $T$. heudelotti $\mathrm{CHCl}_{3}$ fractions showed good anti-staphylococcal activity and MIC values of $\leq 5$ $\mathrm{mg} / \mathrm{ml}$ against multidrug resistant wound isolate of Ps. aeruginosa. The M. tomentosa $\mathrm{CHCl}_{3}$ fractions also showed better activities against E. coli, B. subtilis, $P$. vulgaris and Salmonella enterica var. choleraesius, while that of T. heudelotti gave additional activities against A. haemolyticus and Staphylococcus epidermidis (Table 3).

Aladesanmi and Odediran (2000) had reported the isolation of antimicrobial phenolic acids from the EtOAc fraction of $T$. heudelotti. However, activities at $<5 \mathrm{mg} / \mathrm{ml}$ given by the $\mathrm{CHCl}_{3}$ fraction against $66 \%$ of the multidrug resistant organisms suggest the possibility of some other active constituent(s), whose activities are synergistic with the phenolic acids of the fractions. The frequent occurrence of variations in the MIC values within species and between related organisms suggests that resistances to $M$. tomentosa and $T$. heudelotti, when they occur, are due to intrinsic properties rather than acquired characters of the species. For these reasons, it would be useful if the extracts of the plants or active principles can be exploited for development into antimicrobial chemotherapeutic agents. Similarly there is the need to characterize the active principles in $M$. tomentosa. These plant extracts and fractions may serve as a good source of some cheap and highly effective antimicrobial agents for bacteria infections caused by multiresistant organisms.

The generation of the reactive oxygen species (ROS) beyond what the ability of the body can cope with leads to oxidative stress (Sies 1985, Gutteridge and Halliwell 1994, Maxwell 1995). Free radical oxidative stress has been implicated in the pathogenesis of a variety of human diseases like: artherosclerosis, diabetes mellitus, hypertension, inflammation, cancer and AIDS (Halliwell and Gutteridge 1989). The use of DPPH scavenging assays in assessing the cell membrane integrity/cell membrane stabilising capacities of plant constituents has given explanations as to the possible ways by which phytomedicines could help to reduce diseases caused by infections, inflammation and oxygen radicals generation affecting the cell membrane (Sadique et al 1989, Tsuda, 1998, 2000). The models of scavenging DPPH free radicals used are the rapid screening and the photometric assay methods commonly employed for evaluating antioxidants activities based on their abilities to donate hydrogen ion (Kumazawa, et al 2002). The DPPH is a free radical stable at the room temperature. The methanolic solution gives a purple colouration which when reduced by an antioxidant molecule gives rise to a yellow solution. On comparison with ascorbic acid (AA), a standard antioxidant drug, the antioxidant activities of the extracts in decreasing order were $M$. acuminata $>$ AA $>T$. heudelotti $>E$. senegalensis $>M$. tomentosa $>$ $P$. barteri $>S$. jollyamum. The $\mathrm{EC}_{50}$ values of $M$. acuminata, AA, $T$. heudelotti and E. senegalensis antioxidant activities were higher than that of $14.16 \pm 0.20 \mu \mathrm{g} / \mathrm{ml}$ reported for rutin, a pure standard antioxidant compound (Mensor et al 2001). However, only M. tomentosa and T. heudelotti gave both strong radical scavenging abilities (Table 4) and antimicrobial activities (Tables 2, 3). It is therefore desirable to isolate and characterise the antioxidant agents from these two plants, $T$. heudelotti and $M$. tomentosa and determine whether or not the same constituents are responsible for both the antimicrobial and antioxidant activities.

\section{Conclusion}

The methanolic extracts of E. senegalensis, G. arboreum, M. acuminata, P. zeylanica, P. barteri and $P$. guajava have no antibacterial and antifungal activities. The leaf extracts of M. tomentosa and T. heudelotti showed relatively high antimicrobial activities complemented with impressive antioxidant activities. $M$. acuminata with the highest antioxidant activity showed no antimicrobial activity. The antimicrobial activities were found in the $\mathrm{CHCl}_{3}$ partition fractions of $T$. heudelotti and $M$. tomentosa leaves.The former would be a better choice for antimicrobial activity, the later, M. acuminata leaf, would be good as an antioxidant agent. These results showed that the active plants M. tomentosa and $T$. heudelotts, especially the latter, could be further exploited for chemotherapeutic agents that could be used against infections caused by multiple antibiotic resistance strains very common in Nigeria. Alternatively, the extracts and active fractions of these plants may be used directly in ethnomedicine as antimicrobial agents. 
Adebajo et al., Afr. J. Trad. CAM (2007) 4 (2): 173 - 184

\section{References}

1. Abbiw, D. (1989). Useful plants of Ghana. University of Ghana, Lego and Royal Botanic Gardens,Kew, London, England.

2. Abo, K. A., Ashidi, J. S. (1999). Antimicrobial screening of Bridelia micrantha, Alchornea cordifolia and Boerhavia diffusa. Afri.J.Med. Medical Sci.. 28 (3-4): 167-9.

3. Adegoke, E., Akinsanya, A. and Nagu, A. (1968). Studies of Nigerian medicinal plants I. A preliminary survey of plant alkaloids. J. West Afri. Sci. Assoc.. 13: 13-17.

4. Aderinokun, G.A., Lawoyin, J.O. and Onyeaso, C. O. (1999). Effect of two common Nigerian chewing sticks on gingival health and oral hygiene. Odontostomatol Tropical. 22 (87): 13-8

5. Agrawal, A., Srirvastava, , S., Srivastava, J. N. and Srivastava, M. M. (2004). Inhibitory effect of the plant Boerhavia diffusa Linn against the dermatophytic furgus, Microsponum fulvum. J. Environm Biol.25 (3): 30711

6. Aladesanmi, A. J., Sofowora, A. and Leary, J. D. (1986). Preliminary Biological and Phytochemical Investigation of two Nigerian Medicinal Plants. Int.. J. Crude Drug Res.. 24(3): 147-153.

7. Aladesanmi, A. J., Odediran, S. A. (2000). Antimicrobial activity of Trichilia heudelotti leaves. Fitoterapia. 71: 179-182.

8. Amarnath Satheesh, M. and Pari, L. (2004). Antioxidant effect of Boerhavia diffusa Linn in tissue of alloxan induced diabetic rats. Indian J.Exptl. Biol.. 42 (10): 989-992.

9. Ames B.N, Shigenaga M.K, Hagen T.M(1993). Oxidants, antioxidants and the degenerative diseases of aging. Proc Natl. Acad. Sci. 90:7915-7922.

10. Barrow, G., and Feltham, R. (1993). Cowan and Steel's manual for the identification of medical bacteria, $3^{\text {rd }}$ edition. Cambridge University Press, Cambridge, United Kingdom.

11. Burits, M., and Bucar, F. (2000). Antioxidant activity of Nigella sativa essential oil. Phytother. Res., 14: 323-328.

12. Camporese, A., Balick, M.J., Arrigo, R., Esposito, R. G., Morsellino, N., De Simone, F., and Tubaro, A. (2003). Screening of antibacterial activity of medicinal plants from Belize (Central America). J..Ethnopharmacol. 87: 103-107.

13. Chambers, H. F. (2003). Tracking the spread of CMRSA. Alliance for the prudent use of Antibiotics. APUA Newsletter, 21(2): 1-5.

14. Chopra, I., Hodgson, J., Metcalf, B. and Poste, G. (1997). The search for Antimicrobial Agents Effective against Bacteria Resistant to Multiple Antibiotics. Antimicrobial Agents Chemother., 41(3): 497-503.

15. Chude, M. A., Onzakure, O. E., Afonne, O. S., Gamaliel, K. S., Vonghan, O. H. and Obi, E. (2001). Hypoglycaemic effect of the aqueous extract of_Boerhavia diffusa_leaf. Indian J. Pharmacol., 33: 215-216.

16. Dalziel, J. M. (1956). The useful plants of West Tropical Africa, Crown Agents for Overseas Government, London.

17. Gutteridge, J.M.C and Halliwell, B. (1994) Oxidative stress. In: Antioxidants in Nutrition, Health and Diseases. Oxford University Press. pp $90-102$.

18. Halliwell, B. and Gutteridge, J.M.C (1989) Free radicals in Biology and Medicine $2^{\text {nd }}$ Edition. Clarendon Press, Oxford. pp416 - 494.

19. Igbal, A., Zafar, M., and Faiz, M. (1998). Screening of some Indian Medicinal Plants for their antimicrobial properties. J. Ethnopharmacol. 62: 183-193.

20. Iwata K. (1992). Drug resistance in human pathogenic fungi. Europian J. Epidemiol. 8: 407-421.

21. Kokuraro, J.O. (1993). Medicinal plants of East Africa. $2^{\text {nd }}$ edition. East Africa Literature Bureas, Nairobi.

22. Kumazawa, S., Taniguchi M. Suzuki Y, Shimura M, Kwon M., Nakayama T. (2002). Antioxidant activity of Polyphenols in Carob Pods. .J. Agric. Food Chem. 50: 373-377.

23. Lamikanra, A., Fayinka, S.T. and Olusanya, O.O. (1989). Transfer of low level trimethoprim resistance in faecal isolates obtained from apparently healthy Nigerian students. FEMS Microbiology Letters. 50:275-8.

24. Lemma, H., Debella, A., Addis, G., Kunert, O., Geyid., A., Teka., F. and Yersan, K. (2002). Antibacterial activity of Plumbago zeytanica Linn roots on some pneumonia causing pathogens. SINET: Ethopian J Sci. 25(2): 285-294.

25. Maxwell, S.R.J. (1995) Prospects for the use of antioxdant therapies. Drugs 49, 345 - 361.

26. Mc Vann, A., Harlik, I., Jonbert, P.H. and Monteagudo, F.S. (1992). Cardiac glycoside poisoning involved in deaths from medicines. South Afri Med Journal. 81(3): 139-41.

27. Mensor, Luciana L., Fábio S. Menezes, Gilda G. Leitão, Alexandre S. Reis, Tereza C. dos Santos, Cintia S. Coube, Suzana G. Leitão, (2001) Screening of Brazilian plant extracts for antioxidant activity by the use of DPPH free radical method. Phytother. Res.. 15, 127-130. 
Adebajo et al., Afr. J. Trad. CAM (2007) 4 (2): 173 - 184

28. Michido, D.A. and Ado, S.A. (1999). Antibacterial activity of Anogeissus leiocarpus and Prosopis africana leaf and bark extracts. J. Pharmaceu Res. Develop.. 4(1): 53-56.

29. Moody, J.O. and Roberts, V.A. (2002a). Anti viral effect of selected medicinal plants I. Effect of Diospyros barteri, D. monbuterisis and Sphenoceutrum jollyanum on polioviruses. Nig. J. Nat. Prod.Med. 6:4-6.

30. Moody, J. O. and Roberts, V. A. (2002b). Anti viral activities of selected medicinal plants II. Effects of extracts of Diospyros barteri, D. monbuterisis and Sphenoceutrum jollyanum on cowpea mosaic viruses: Pharmaceutical Biol. 40(5): 342-345.

31. Murray, P. R., Baron, E. J., Pfaller, M. A., Tenover, F. C., Yolke, R. H. (1995). Manual of Clinical Microbiology, $6^{\text {th }}$ edition ASM, Washington, DC

32. Mutho, K.N., Ohiri, P.C., Ezugwu, C. O. (1998), Antipyretic and analgesic activities of Sphenocentrum jollyanum. Nig. J. Nat.Prod. Med.. 2: 52-53.

33. National Committee for Clinical Laboratory Standards. (2003). Performance Standards for Antimicrobial Disk Susceptibility Tests; Approved Standards-Eighth Edition Volume 23 No1, M2-A8.

34. Okafor, J.I., Eze, E.A. and Njoku, O.U. (2001). Antifungal activities of the leaves of Baphia nitida, Cassia alata, Ficus exasperata and Gossypium arboretum. (2001). Nig. J. Natural Products and Medicine. 5: 59-60.

35. Oke, J.M. and Hamburger, M.O. (2002). Screening of some Nigerian Medicinal Plants for antioxidant activity using 2,2, diphenyl-picyl-hydrazyl radical. Afr. J. Biomedical Res. 5: 77-79.

36. Okeke, I.N. and Sosa, A. (2003). Antibiotic resistance in Africa - discerning the enemy and plotting a defence. Africa Health, (March 2003): 10-15

37. Oliver-Bever, B. (1986). Medicinal plants in Tropical West Africa, Cambridge University Press, Great Britain, pp.375.

38. Olukoya, D.K., Idika and Odugbemi, T. (1993). Antibacterial activity of some medicinal plants from Nigeria. J. Ethnopharmacol. 39: 69-72.

39. Oluronke, T., Hong-xi, X.U. and Song, F. L. (1999). Antibacterial activities of extracts from Nigerian chewing sticks. Phytother. Res. 13(8); 675-679.

40. Reeves, D. S., Phillips, I., and Williams, J. D. (1979). Laboratory Methods in Antimicrobial Chemotherapy. Longman Group Ltd, Edinburgh. p. 20.

41. Sadique J, Al-Rqobab NA, Bughaith MF, El-Gindy AR (1989): The bioactivity of certain medicinal plants on the stabilization of RBC membrane system. Fitoterapia. LX: 525-532.

42. Sanchez-Moreno, C., Larrauri, J. A. and Saura-Calixto, F. (1998). A procedure to measure the antiradical efficiency of polyphenols. J. Sci. Food Agric. 76(2):270-276.

43. Sies, H. (1985) Oxidative stress: Introductory remarks. In: Sies, H, (Ed.), Oxidative Stress. Academic Press, Orlando, FL, pp. 1-8.

44. Sofowora, A. (1993). Medicinal plants and Traditional Medicine in Africa. Spectrum Books Limited.

45. Tsuda T. (1998) Dietary cyanidin 3-0-beta-D-glucoside increases ex vivo oxidative resistance of serum in rats. Lipids 33:583-588.

46. Tsuda T (2000): The role of anthocyanins as an antioxidant under oxidative stress in rats. Biofactors; 13:133-139.

47. Wu C.D.; Darout I.A.; Skaug N. (2001). Chewing sticks: timeless natural toothbrushes for oral cleansing. J. Periodontal Res. 36(5); 275-284. 